JOURNAL OF

SYNCHROTRON

RADIATION

ISSN 1600-5775

Received 9 August 2021

Accepted 28 December 2021

Edited by Y. Amemiya, University of Tokyo, Japan

Keywords: fluoride; hydroxyapatite; root caries; remineralization; synchrotron radiation.
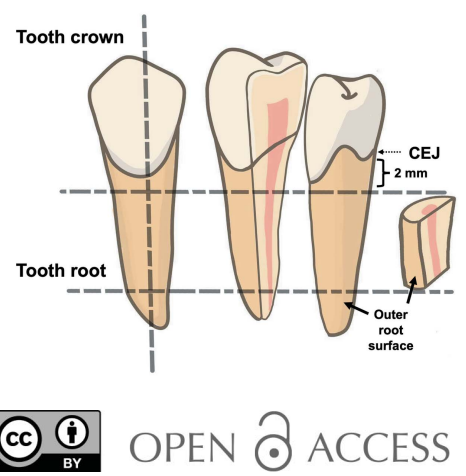

\section{Synchrotron radiation analysis of root dentin: the roles of fluoride and calcium ions in hydroxyapatite remineralization}

\author{
Nutthapong Kantrong, ${ }^{\mathrm{a}, \mathrm{b}, \mathrm{c} *}$ Krassawan Khongkhaphet, ${ }^{\mathrm{c}}$ Nutnicha Sitornsud, \\ Pakaporn Lo-apirukkul, ${ }^{c}$ Waraporn Phanprom, ${ }^{\mathrm{b}, \mathrm{c}}$ Catleya Rojviriya, ${ }^{\mathrm{d}}$ \\ Penphitcha Amonpattaratkit ${ }^{\mathrm{d}}$ and Watcharaphong Ariyakriangkai ${ }^{\mathrm{e}}$
}

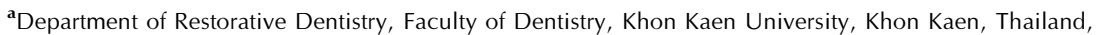
${ }^{\mathbf{b}}$ Oral Biology Research Unit, Faculty of Dentistry, Khon Kaen University, Khon Kaen, Thailand, ${ }^{\mathbf{c}}$ Research Group of Chronic Inflammatory Oral Diseases and Systemic Disease Associated with Oral Health, Faculty of Dentistry, Khon Kaen University, Khon Kaen, Thailand, ${ }^{\mathbf{d}}$ Synchrotron Light Research Institute, Nakhon Ratchasima, Thailand, and ' Department of Restorative Dentistry and Periodontology, Faculty of Dentistry, Chiang Mai University, Chiang Mai, Thailand. *Correspondence e-mail: natthkan@kku.ac.th

Although the use of fluoride for root caries control is reported to be effective, the mechanism of maintaining hydroxyapatite is still unclear. This study elucidates the roles of fluoride in the recrystallization of hydroxyapatite, and the impact of calcium to maintain the abundance of hydroxyapatite on acidchallenged root dentin with a novel approach - using synchrotron radiation. Root dentin samples obtained from 40 extracted human premolars were subjected to $\mathrm{pH}$ challenge in combination with fluoride treatment. The effect of fluoride on hydroxyapatite regeneration on the root was investigated by using a range of fluoride concentrations (1000-5000 p.p.m.) and the EDTA-chelation technique in vitro. Synchrotron radiation X-ray micro-computed tomography and X-ray absorption spectroscopy were utilized to characterize the chemical composition of calcium species on the surface of prepared samples. The percentage of hydroxyapatite and the relative abundance of calcium species were subsequently compared between groups. The absence of calcium or fluoride prevented the complete remineralization of hydroxyapatite on the surface of early root caries. Different concentrations of fluoride exposure did not affect the relative abundance of hydroxyapatite. Sufficient potency of 1000 p.p.m. fluoride solution in promoting hydroxyapatite structural recrystallization on the root was demonstrated. Both calcium and fluoride ions are prerequisites in a caries-prone environment. Orchestration of $\mathrm{F}^{-}$and $\mathrm{Ca}^{2+}$ is required for structural homeostasis of root dentin during acid attack. Sustainable levels of $\mathrm{F}^{-}$and $\mathrm{Ca}^{2+}$ might thus be a strict requirement in the saliva of the population prone to root caries. Fluoride and calcium contribute to structural homeostasis of tooth root, highlighting that routine fluoride use in combination with calcium replenishment is recommended for maintaining dental health. This study also demonstrates that utilization of synchrotron radiation could provide a promising experimental platform for laboratory investigation especially in the dental material research field.

\section{Introduction}

A higher prevalence of root caries has been reported as the number and proportion of people over the age of 65 increases across the globe (Heasman et al., 2017; UN, 2019). Considering the causative factors contributing to root caries, such disease is caused by an imbalanced relationship between remineralization and demineralization on the tooth surface (BeltránAguilar et al., 2005; Young et al., 2015), a state of which an exchange of calcium, phosphate and fluoride is involved to 
achieve a saturation point (Neel et al., 2016). The root portion is also easily approached by cariogenic stimuli due to the thinness of the cementum layer (Dastmalchi et al., 1990), which puts the underlying dentin at risk of mineral loss. Notably, a previous report has suggested that the formation of fluorapatite during acid attack is not considered as a remineralization since the process involves mineral gain that rather retards further demineralization (Cury \& Tenuta, 2009). Structural deterioration of the tooth root resulting from a chronic polymicrobial infection-induced demineralization process (Young et al., 2015) thus requires careful structural characterization for a better understanding of the mechanisms of hydroxyapatite dissolution where minimally invasive therapy could be implemented for root caries prevention.

Tooth is a hard tissue primarily composed of enamel and dentin with hydroxyapatite as the main inorganic component (Zipkin, 1970). During the formation of hydroxyapatite, a diversity of calcium-containing precursors exists for its nucleation, i.e. dicalcium phosphate $\left(\mathrm{CaHPO}_{4}\right), \alpha$ - and $\beta$ tricalcium phosphate $\left[\mathrm{TCP} ; \mathrm{Ca}_{3}\left(\mathrm{PO}_{4}\right)_{2}\right]$. The availability of such precursors allows crystallization of hydroxyapatite to occur (Francis \& Webb, 1970; Blumenthal \& Posner, 1973). In addition, it has been reported that synthesis of hydroxyapatite is achieved by incorporating calcium carbonate $\left(\mathrm{CaCO}_{3}\right)$ and calcium oxide $(\mathrm{CaO})$ in the chemical interactions (SobczakKupiec \& Wzorek, 2012; Kim \& Ohtsuki, 2016; Habibah \& Salisbury, 2020). Notably, hydroxyapatite is a form of crystalline calcium phosphate with the formula $\mathrm{Ca}_{10}\left(\mathrm{PO}_{4}\right)_{6}(\mathrm{OH})_{2}$, found in both teeth and bone with slight differences in lattice parameters, crystal size, crystallinity and composition (LeGeros, 1990).

The use of fluoride has been strongly proven to be effective in preventing dental caries in permanent teeth (SBU, 2002). Since carious lesions progress more rapidly in dentin than in enamel due to a higher critical $\mathrm{pH}$ for dentin and dentin is thus more susceptible to acid dissolution (Kantrong et al., 2021), prevention of initial root caries has been of utmost importance. In addition, systematic reviews have shown that dentifrice containing 5000 p.p.m. fluoride is more efficient in reducing active root carious lesions when compared with 1100 to 1450 p.p.m. dentifrice, especially for elderly people with exposed root surfaces (Wierichs \& Meyer-Lueckel, 2015). The use of 5000 p.p.m. fluoride toothpaste or gel in preventing root caries has been corroborated by several other investigations (Wierichs \& Meyer-Lueckel, 2015; Slayton et al., 2018). Most studies have quantified the change in lesion depth, surface microhardness, mineral loss and maximum mineral density due to different levels of fluoride exposure on carious lesions (Shahmoradi et al., 2014). However, deposition of calciumbased biominerals deposited on the root dentin surface by fluoride is yet unclear. Structural characterization of hydroxyapatite regenerated after fluoride application on root dentin hence remains to be investigated.

Previous studies have suggested a prerequisite of free calcium ions $\left(\mathrm{Ca}^{2+}\right)$ in the oral microenvironment for the regeneration of hydroxyapatite crystals (Reynolds, 2008; Chaudhary et al., 2017). Concentrated fluoride promotes precipitation of calcium and phosphorus ions over acidinduced early root caries (Kantrong et al., 2021), possibly suggesting that an ample amount of fluoride is needed for root dentin remineralization. Although the crystalline hydroxyapatite in the demineralized and remineralized enamel have been characterized using synchrotron radiation (Asaizumi et al., 2017; Sui et al., 2018; Tanaka et al., 2010), the dynamic change of hydroxyapatite and its precursors under acidic circumstances is still unclear, and whether $\mathrm{Ca}^{2+}$ and fluoride ions $\left(\mathrm{F}^{-}\right)$contribute to the hydroxyapatite remineralization on the root dentin requires further investigation. Thus, the objectives of this study were to determine the role of fluoride and calcium in hydroxyapatite remineralization on early root caries by investigating the gradients of calcium species required for hydroxyapatite remineralization. Our analysis implemented using synchrotron radiation as a novel technique to gain a better understanding of how fluoride and calcium ions orchestrate the remineralization of hydroxyapatite on root dentin.

\section{Materials and methods}

\subsection{Preparation of root dentin samples}

This study was executed under an ethical approval granted by the Ethics Committee for Human Research of Khon Kaen University, based on the declaration of Helsinki (Approval number: HE632048). Briefly, 40 sound human premolar teeth, extracted for orthodontic reasons and stored in $0.1 \%$ thymol solution, were cleaned of any residual gingival tissue and cementum using a scalpel blade (No.15). The teeth were sectioned transversely at a distance of $2 \mathrm{~mm}$ underneath the cemento-enamel junction (CEJ) to remove the crown and then longitudinally to produce $4 \mathrm{~mm} \times 4 \mathrm{~mm} \times 2 \mathrm{~mm}$ root sections (Fig. 1) using a precision cutting machine with incorporated water coolant (Mecatome T180 PRESI; Eybens, France). Samples ( $N=5$ per group) were randomly assigned to eight groups as follows:

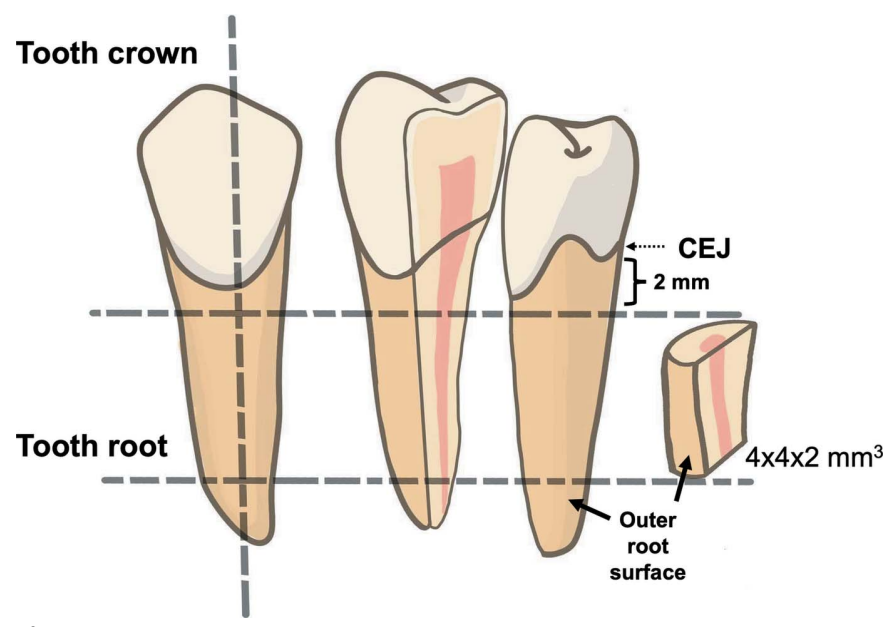

Figure 1

Sectioning of a human premolar to obtain a root dentin specimen for further analysis. 
Group $A$ was immersed in deionized water for 11 days serving as the experimental baseline.

Group $B$ was immersed in a demineralization solution (2.2 $\mathrm{m} M \mathrm{CaCl}_{2}, 2.2 \mathrm{mM} \mathrm{KH}_{2} \mathrm{PO}_{4}, 0.05 M$ acetic acid, $\mathrm{pH} 4.7$ ) for four days to produce early root carious lesions and then immersed in deionized water for the following ten days.

Groups $C, D, E$ and $F$ were immersed in a demineralized solution for four days to produce early carious lesions and subjected to a ten-day $\mathrm{pH}$-cycling using the demineralization solution and remineralization solution (1.5 $\mathrm{mM} \mathrm{CaCl}_{2}$, $0.9 \mathrm{~m} M \mathrm{NaH}_{2} \mathrm{PO}_{4}, 0.15 M \mathrm{KCl}, \mathrm{pH}$ 7) along with fluoride treatment solutions containing 0 p.p.m. (Group $C)$, 1000 p.p.m. (Group D), 1450 p.p.m. (Group E) and 5000 p.p.m. fluoride (Group $F$ ).

Groups $G$ and $H$ were subject to the same pH-cycling protocol with an addition of $200 \mu \mathrm{L}$ of $17 \%$ ethylenediamine tetraacetic acid (EDTA) to the remineralization solution in order to chelate free $\mathrm{Ca}^{2+}$, and treated with 1450 p.p.m. (Group G: 1450 p.p.m. $\mathrm{F}^{-} /$EDTA) or 0 p.p.m. fluoride solutions (Group H: 0 p.p.m. F ${ }^{-} /$EDTA). $^{2}$

\section{2. $\mathrm{pH}$ challenge, treatment with fluoride solution and chelation of $\mathrm{Ca}^{2+}$ from remineralization solution}

The $\mathrm{pH}$-cycling was scheduled for ten days in a $37^{\circ} \mathrm{C}$ environment comprising the following phases (Fig. 2): $3 \mathrm{~h}$ of demineralization twice a day; 5 min sodium fluoride $(\mathrm{NaF})$ solution treatment after each demineralization phase; $2 \mathrm{~h}$ of remineralization in between the demineralization phases; and a $16 \mathrm{~h}$ overnight period of remineralization.

Treatments were given after each $3 \mathrm{~h}$ demineralization period for $5 \mathrm{~min}$. Specimens were immersed in an $\mathrm{NaF}$ solution prepared by thoroughly mixing $\mathrm{NaF}$ powder (Ricca Chemical, Texas, USA) with the remineralization solution, followed by centrifugation at 100 r.p.m. for $5 \mathrm{~min}$ at room temperature. All solutions were prepared daily, and the $\mathrm{pH}$ of each solution was monitored to ensure experimental consistency.

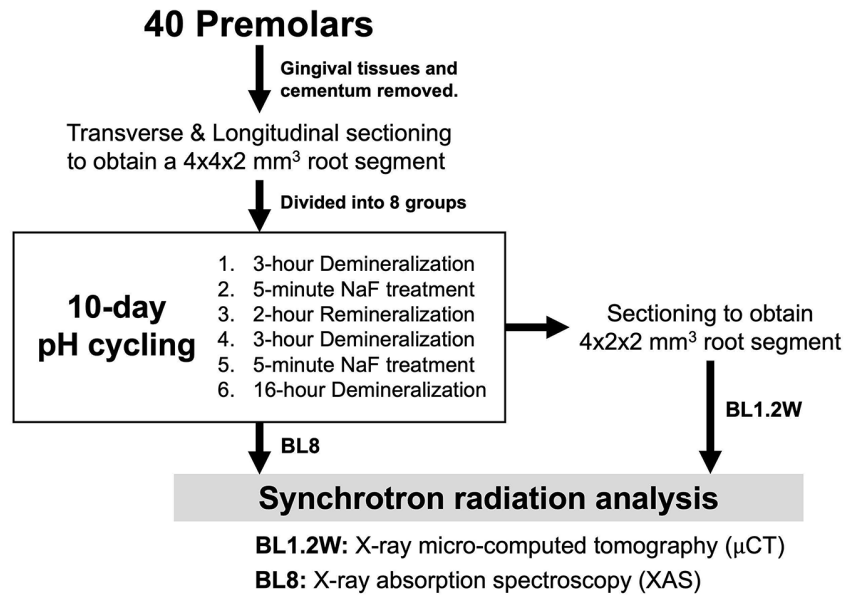

Figure 2

Schematic depiction of laboratory procedures performed in this experimental study.
In this study, fluoride solution was chosen for experimental execution as opposed to a dentifrice slurry due to its more precise preparation to obtain the required concentration of fluoride used in a mock experimental treatment. A complexometric titration of remineralization solution with $17 \%$ EDTA was used to remove unbound $\mathrm{Ca}^{2+}$ to simulate a salivary microenvironment lacking unbound $\mathrm{Ca}^{2+}$. The EDTA titration technique was introduced in the early 1960s with the main purpose to determine the calcium level in water and using an Eriochrome Black $\mathrm{T}$ as an indicator of the photometric method (Lacy, 1963; Flaschka \& Ganchoff, 1961). When EDTA formed a complex with $\mathrm{Ca}^{2+}$, the solution immediately changed color from purple to blue as indicated by the Eriochrome Black T indicator (Aquacheme Co. Ltd., Bangkok, Thailand) (Fig. 3). $\mathrm{A} \mathrm{Ca}^{2+}$ chelating end-point was achieved when the remineralization solution became dark blue in color, reflecting the absence of free $\mathrm{Ca}^{2+}$. After pH-cycling was completed, the samples were immersed in deionized water; root dentin samples were further dried for $24 \mathrm{~h}$ prior to analysis.

Given that the binding ratio of $\mathrm{Ca}^{2+}$ to EDTA is $1: 1$ (Keowmaneechai \& McClements, 2002), $188.5 \mu \mathrm{L}$ of $17 \%$ EDTA was needed for a complete Ca-EDTA chelation in $100 \mathrm{ml}$ remineralization solution. During our preliminary test with complexometric titration, we determined the volume of EDTA used for direct titration and a $200 \mu \mathrm{l}$ volume of $17 \%$ EDTA was sufficient for chelating free $\mathrm{Ca}^{2+}$. Eriochrome Black $\mathrm{T}$ turned saturated dark blue indicating a complete
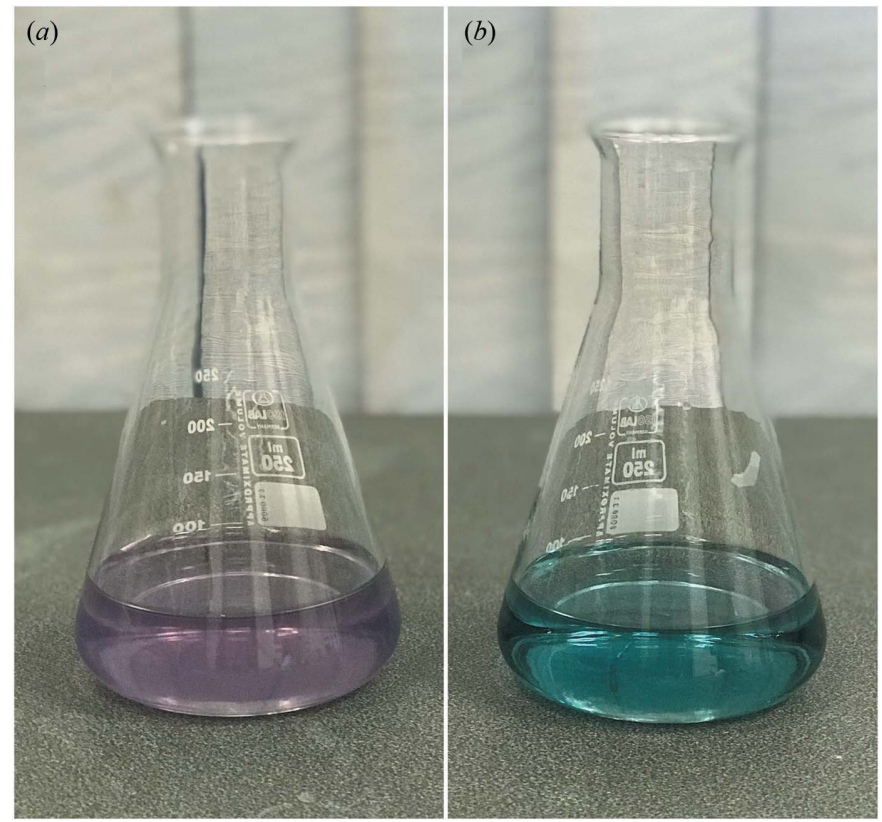

Figure 3

Complexometric titration of free $\mathrm{Ca}^{2+}$ in the remineralization solution using $17 \%$ EDTA. Note that the colorless remineralization solution turned purple when Eriochrome Black T formed a complex with unbound $\mathrm{Ca}^{2+}(a)$. However, when $200 \mu \mathrm{l}$ of $17 \%$ EDTA was added, unbound $\mathrm{Ca}^{2+}$ formed a complex with EDTA instead and the solution hence immediately turned blue as indicated by Eriochrome Black T. The saturation point of dark blue color indicates the absence of free $\mathrm{Ca}^{2+}$ available in the remineralization solution $(b)$. 
chemical binding of free $\mathrm{Ca}^{2+}$ with EDTA. A volume of $200 \mu \mathrm{l}$ EDTA in combination with Eriochrome Black Twas thus used for freshly preparing the reagents used for the remineralization phase.

\subsection{Synchrotron radiation X-ray micro-computed tomography}

Synchrotron radiation X-ray micro-computed tomography (micro-CT, $\mu \mathrm{CT}$ ) was used in this study to non-invasively characterize the three-dimensional structure of dentin specimens $(4 \mathrm{~mm} \times 2 \mathrm{~mm} \times 2 \mathrm{~mm})$, and was carried out at beamline BL1.2W at the Synchrotron Light Research Institute (SLRI), Nakhon Ratchasima, Thailand. X-ray radiation was generated from a $2.2 \mathrm{~T}$ multipole wiggler at the $1.2 \mathrm{GeV}$ Siam Photon Source. Each specimen was stabilized in a polyimide tube mounted on a rotary stage. A total of $900 \mathrm{X}$-ray projections were collected for $180^{\circ}$ rotation with a step of $0.2^{\circ}$. Images were obtained with a filtered polychromatic X-ray beam at a mean energy of $14 \mathrm{keV}$ and $32 \mathrm{~mm}$ distance from the source. All tomographic scans were acquired at a pixel size of $1.44 \mu \mathrm{m}$ via the detection system equipped with a $200 \mu \mathrm{m}$ thick YAG:Ce scintillator, white-beam microscope (Optique Peter, France $)$ and pco.edge $5.5 \mathrm{sCMOS}$ camera $(2560 \times 2160$ pixels, 16 bits). Data processing and tomographic reconstruction were performed using Octopus Reconstruction software (Tescan Orsay Holding, Brno, Czech Republic). The 3D visualization of acid-induced root caries was performed using Drishti software, Version 2.6 (ANU Vizlab, Canberra, Australia).

\subsection{Synchrotron radiation analysis of X-ray absorption near-edge structure (XANES)}

The calcium $K$-edge was used to characterize the chemical structure of calcium species in carious root dentin using X-ray absortion spectroscoopy (XAS) techniques. The Ca $K$-edge spectra were acquired using fluorescent-yield mode at XAS beamline BL8 of SLRI. The fluorescent-yield spectra of the Ca $K$-edge were obtained from three regions with the following energy ranges and energy steps: 3938.5 to $4018.5 \mathrm{eV}$ (5 eV step), 4018.5 to $4118.5 \mathrm{eV}(0.2 \mathrm{eV}$ step) and 4118.5 to $4238.5 \mathrm{eV}$ ( $5 \mathrm{eV}$ step). The dwell time for each step across all energy regions of samples was $1 \mathrm{~s}$. The fluorescence-yield spectrum was converted to absorption using the following equation (1), routinely used at BL8 SLRI,

$$
\frac{I_{\mathrm{f}}}{I_{0}}=\frac{\Omega}{4 \pi}\left[\frac{\mu(E)}{\mu(E)+\mu_{\mathrm{bkg}}(E)+\mu_{\mathrm{tot}}\left(E_{\mathrm{f}}\right)\left(\sin \theta / \sin \theta_{\mathrm{f}}\right)}\right],
$$

where $I_{0}$ is the intensity of the incident X-ray beam, $I_{\mathrm{f}}$ is the intensity of the monitored fluorescence spectrum, $\Omega$ is the solid angle of the detector, $E$ is the X-ray energy, $E_{\mathrm{f}}$ is the energy of the fluorescent $\mathrm{X}$-ray, $\mu(E)$ is the absorption from the tested element, $\mu_{\mathrm{bkg}}(E)$ is the absorption from the background, $\mu_{\mathrm{tot}}(E)$ is the total absorption in the sample, $\theta$ is the angle of the X-ray on the sample, and $\theta_{\mathrm{f}}$ is the the angle of emission of the fluorescent X-rays from the sample.
The examined compounds were Ca-based species usually found in hard tissue including hydroxyapatite, $\mathrm{CaHPO}_{4}$, $\alpha$-TCP, $\beta$-TCP, $\mathrm{CaCO}_{3}$ and $\mathrm{CaO}$ (Sigma-aldrich, St Louis, $\mathrm{MO}$, USA). The detector used for XAS measurement was a 13element germanium detector (GeD; Canberra Ultra-LEGe). A digital X-ray processor (XIA DXP-XMAP) on a National Instruments (PXI-1042) crate was used for counting X-ray photons from $\mathrm{GeD}$ channels as previously described (Klysubun et al., 2012). A distinctive absorption edge of each reference compound was used to identify the composition of calcium species in the samples. The absorption spectra were normalized to remove variations between samples. Combinatorial linear combination fitting (LCF) analysis was performed to determine the relative abundance of hydroxyapatite and other calcium species by Athena software (Ravel \& Newville, $2005)$ in the energy range $4018.5-4118.5 \mathrm{eV}$.

\subsection{Statistical analysis}

The mean percentage difference of hydroxyapatite was analyzed using a One-way ANOVA with Bonferroni post hoc test for multiple comparison among groups. Similar to a previous report (Kantrong et al., 2021), we utilized the Shannon diversity index which indicates the clustering of specific calcium species on the root to elucidate the role of $\mathrm{F}^{-}$and $\mathrm{Ca}^{2+}$ in the environments in hydroxyapatite remineralization. Equation (2) was utilized to calculate the Shannon diversity index (Table 1). Statistical analyses were performed using GraphPad Prism, Version 9 (GraphPad Software Inc., La Jolla, CA, USA). A $P$-value of less than 0.05 was considered statistically significant:

$$
\text { Shannon diversity index }\left(H^{\prime}\right)=\sum_{i=1}^{S} p_{i} \ln p_{i}
$$

where $S$ is the total number of calcium species detected on the surface of the root dentin, and $p_{i}$ is the proportion of the $i$ th calcium species as calculated by dividing the number of the $i$ th calcium species by the total number of calcium species detected on the surface of the root dentin.

\section{Results}

\subsection{Characterization of carious root dentin}

The formation of early carious root dentin was characterized by using synchrotron radiation $\mu \mathrm{CT}$. The tomographic volume of the dentin specimens was visualized and analyzed in three dimensions using Drishti software. Color gradients were assigned according to the voxel density of the dentin specimen, in which darker colors represent higher density voxels and lighter colors represent lower density voxels. A thin uniform superficial layer, shown in cyan in Fig. 4, was formed at the surface of root dentin in the acid-induced group. We also noticed a thickening dark band demineralized area on the root dentin when observed under a polarized light microscope (data not shown), indicating the formation of acid-induced early root carious lesions. This result confirmed the process of demineralization at the surface of the specimen by our caries 
Table 1

The percentage and standard deviation of investigated calcium species in each treatment group and calculated Shannon diversity index.

\begin{tabular}{|c|c|c|c|c|c|c|c|c|}
\hline Intervention & Hydroxyapatite & $\alpha$-ТCP & $\beta$-ТCР & $\mathrm{CaHPO}_{4}$ & $\mathrm{CaCO}_{3}$ & $\mathrm{CaO}$ & $R$-factor & $\begin{array}{l}\text { Shannon } \\
\text { diversity } \\
\text { index }\left(H^{\prime}\right)\end{array}$ \\
\hline A: Aged root dentin & $78.2 \pm 3.7$ & 0 & 0 & $21.8 \pm 2.4$ & 0 & 0 & 0.041559 & 0.5244 \\
\hline B: Acid-induced & $73.3 \pm 3.8$ & 0 & 0 & $26.7 \pm 6.3$ & 0 & 0 & 0.007244 & 0.5803 \\
\hline C: F 0 p.p.m. & $75.8 \pm 4.9$ & $15.4 \pm 4.0$ & 0 & 0 & 0 & $8.8 \pm 1.8$ & 0.0038061 & 0.7120 \\
\hline D: F 1000 p.p.m. & $95.0 \pm 2.6$ & 0 & 0 & $5.0 \pm 5.3$ & 0 & 0 & 0.0045462 & 0.1985 \\
\hline E: F 1450 p.p.m. & $91.3 \pm 1.8$ & 0 & 0 & $8.7 \pm 4.0$ & 0 & 0 & 0.0021873 & 0.2955 \\
\hline F: F 5000 p.p.m. & $92.8 \pm 2.0$ & 0 & 0 & $7.2 \pm 2.0$ & 0 & 0 & 0.0033991 & 0.2588 \\
\hline G: F 1450 p.p.m./EDTA & $70.2 \pm 2.3$ & $21.6 \pm 3.8$ & 0 & $4.5 \pm 5.8$ & 0 & $3.7 \pm 1.5$ & 0.0020471 & 0.8409 \\
\hline H: F 0 p.p.m./EDTA & $69.3 \pm 3.3$ & 0 & $7.4 \pm 2.1$ & $15.2 \pm 3.0$ & 0 & $8.1 \pm 5.5$ & 0.0015962 & 0.9367 \\
\hline
\end{tabular}

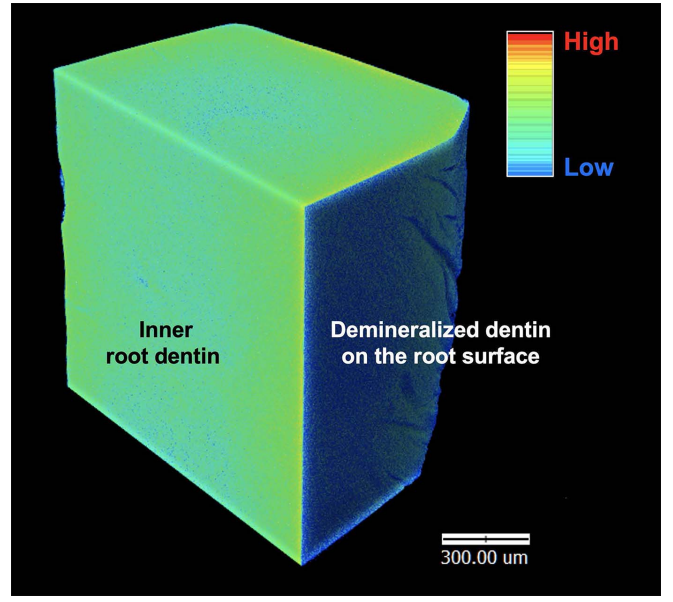

Figure 4

$\mu \mathrm{CT}$ visualization of the demineralized area in the acid-induced group. Induction of surface mineral loss by acidic environment. Colors were assigned according to the density values of each voxel, with higher density values represented by darker colors and low density values by lighter colors.

induction protocol. In addition, $\mu \mathrm{CT}$ analysis revealed a dense band of minerals deposited on the root dentin when exposed to fluoride solution, particularly when 1450 p.p.m. and 5000 p.p.m. were used (Fig. 5).

\subsection{Hydroxyapatite is the main component of root dentin}

Characterization of the mineral composition of the samples was performed by the acquisition of XANES [Fig. 6(a)] using XAS techniques. The structures in the absorption edge of the samples were compared with known spectra of calcium species in human hard tissue. We found that the white line and shoulder of the aged root dentin samples strongly resembled the features found in the absorption edge of hydroxyapatite [Fig. 6(b)]. LCF was subsequently performed to determine the most likely chemical composition of each root dentin specimen. We found that aged root dentin is composed of $78.2 \%$ hydroxyapatite and $21.8 \% \mathrm{CaHPO}_{4}$ (Fig. 7, Table 1). The $R$-factor value, which indicates the fit of the LCF analysis, was in the range $<0.002-0.04$ (Table 1), suggesting a high fitting quality. This reaffirmed that hydroxyapatite is the main native calcium species detected on root dentin.

\subsection{Fluoride promotes hydroxyapatite recrystallization on root dentin}

In this study, we sought to determine whether fluoride solutions affected the remineralization pattern of calcium species on carious root lesions. XAS results indicate that a statistically significant difference $(P$-value $<0.0001)$ of $73.30 \%$ relative abundance of hydroxyapatite in the 0 p.p.m. fluoride

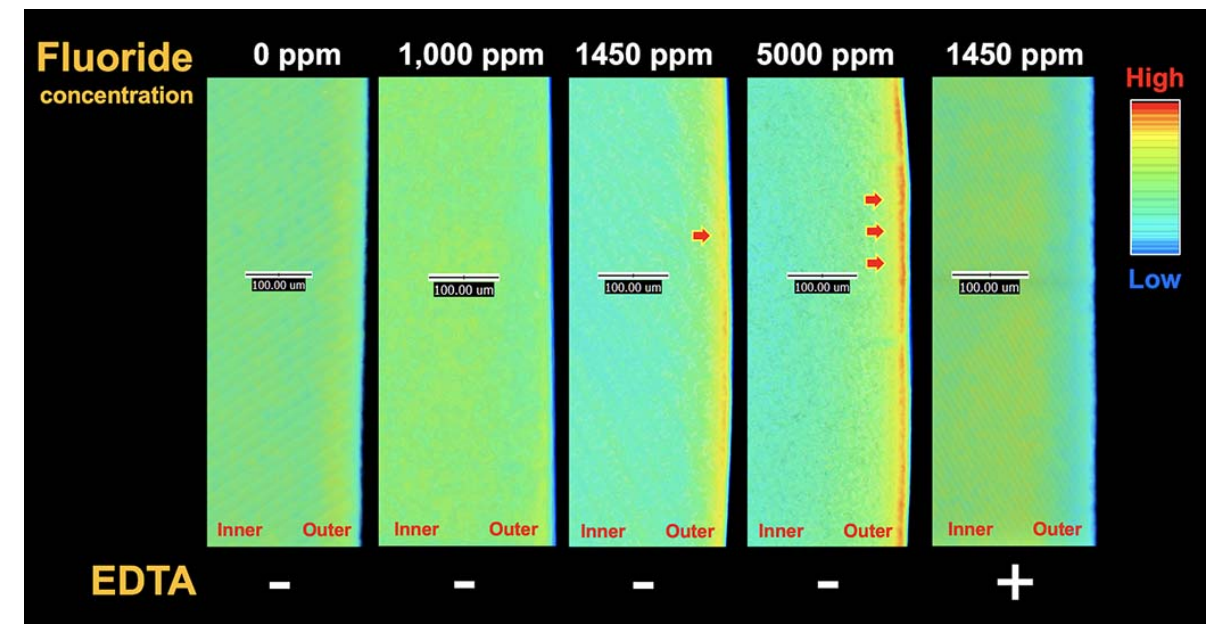

Figure 5

$\mu \mathrm{CT}$ image of superficial mineral deposition on root dentin. Mineral gain on the superficial layer of fluoride-treated samples is clearly seen in a dosedependent manner as indicated by red arrows. 

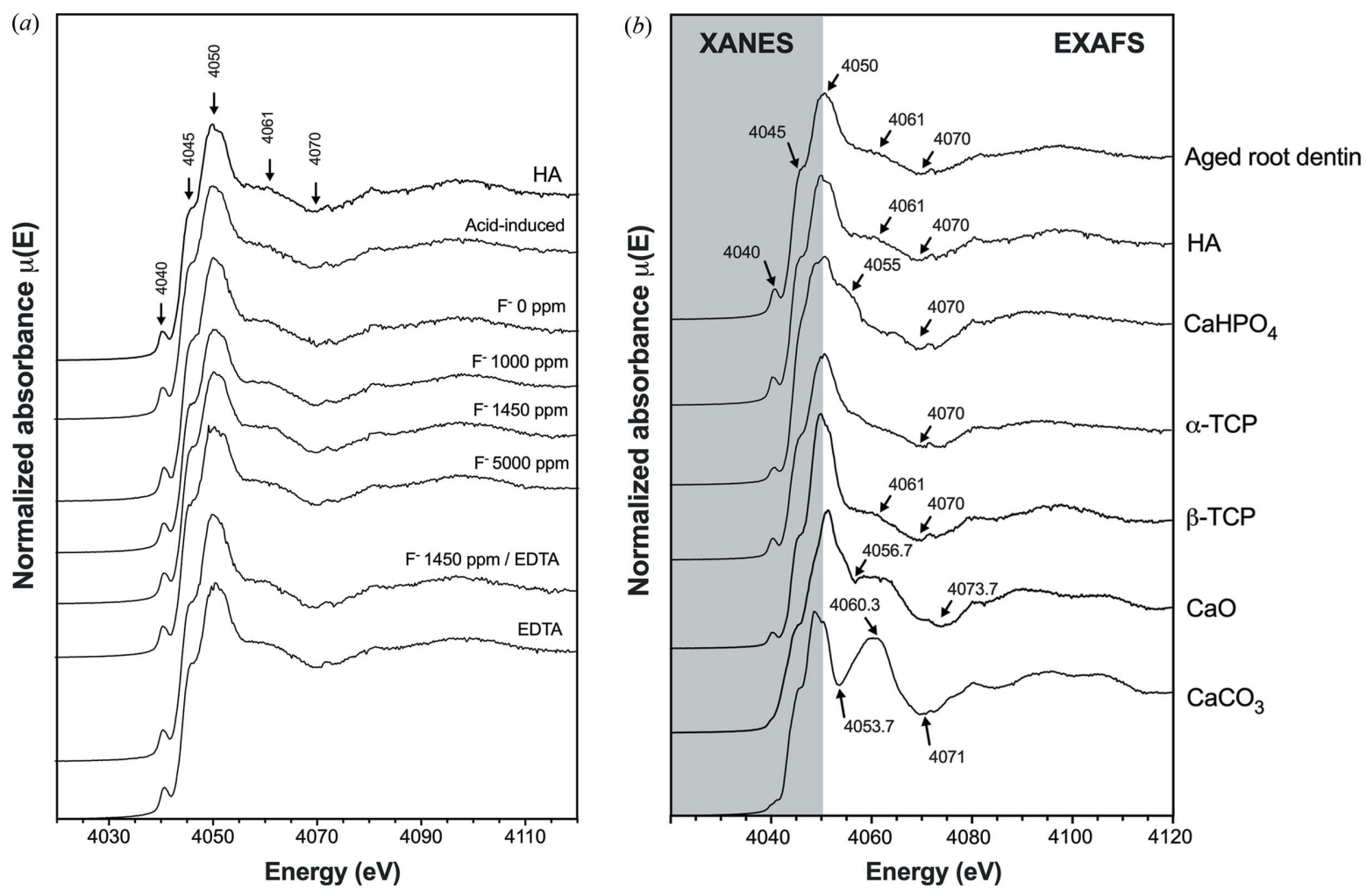

Figure 6

Spectral features of XAS consisting of X-ray absorption near-edge structure (XANES) specifically used for structural analysis and extended X-ray absorption fine structure (EXAFS). (a) The Ca-absorption edge obtained for each treatment group. $(b)$ The structures in the absorption edge of the aged root dentin group strongly resemble the features found in the absorption edge of hydroxyapatite, indicating that hydroxyapatite is the main calcium species found in aged root dentin.

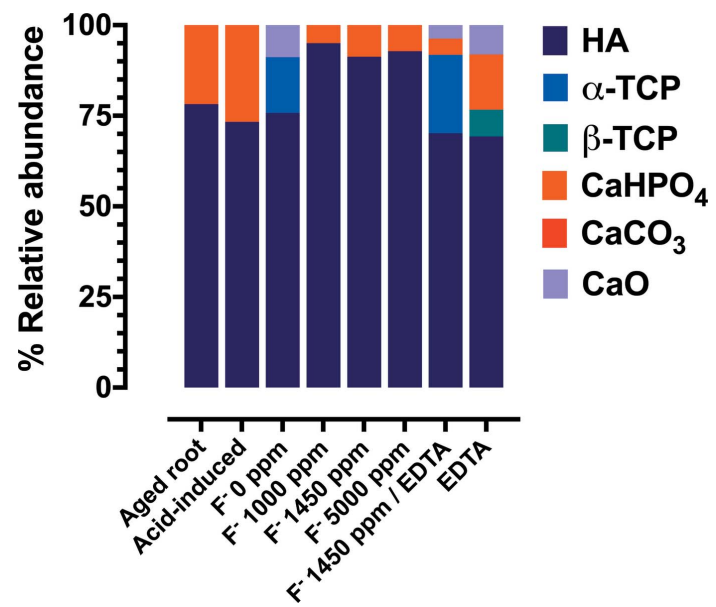

Figure 7

The diversity shift of calcium derivatives in the presence of $\mathrm{F}^{-}$and $\mathrm{Ca}^{2+}$ in the environment. Relative abundance (\%) of the following calcium species: hydroxyapatite, $\alpha$-TCP, $\beta$-TCP, $\mathrm{CaHPO}_{4}, \mathrm{CaCO}_{3}, \mathrm{CaO}$ found in each treatment group.

group compared with $95.0 \%$ in the 1000 p.p.m. fluoride group was present (Fig. 7, Table 2). It is worth noting that the diversity of calcium species found in the treated root dentin were increased in the absence of $\mathrm{F}^{-}$, as indicated by the Shannon diversity index, when comparing the fluorideuntreated group (0 p.p.m. fluoride, $\left.H^{\prime}=0.7120\right)$ with the fluoride-treated group (1000 p.p.m., $H^{\prime}=0.1985$; 1450 p.p.m.,
Table 2

Comparison of the mean of hydroxyapatite percentage in terms of relative abundance for each treatment group.

Asterisks $(*)$ indicate a significant difference. $\mathrm{Ns}=$ non-significant.

\begin{tabular}{llll}
\hline Intervention & $P$-value & Summary & Group \\
\hline Acid-induced / F 0 p.p.m. & 0.0580 & Ns & *B-C \\
Acid-induced / F 1000 p.p.m. & $<0.0001$ & Significant & *B-D \\
Acid-induced / F 1450 p.p.m. & $<0.0001$ & Significant & *B-E \\
Acid-induced / F 5000 p.p.m. & $<0.0001$ & Significant & *B-F \\
Acid-induced / F 1450 p.p.m. with EDTA & $>0.9999$ & Ns & B-G \\
Acid-induced / F 0 p.p.m. with EDTA & $>0.9999$ & Ns & B-H \\
F 0 p.p.m. / F 1000 p.p.m. & $<0.0001$ & Significant & *C-D \\
F 0 p.p.m. / F 1450 p.p.m. & $<0.0001$ & Significant & *C-E \\
F 0 p.p.m. / F 5000 p.p.m. & $<0.0001$ & Significant & *C-F \\
F 0 p.p.m. / F 1450 p.p.m. with EDTA & 0.1798 & Ns & C-G \\
F 0 p.p.m. / F 0 p.p.m. with EDTA & 0.0580 & Ns & C-H \\
F 1000 p.p.m. / F 1450 p.p.m. & $>0.9999$ & Ns & D-E \\
F 1000 p.p.m. / F 5000 p.p.m. & $>0.9999$ & Ns & D-F \\
F 1000 p.p.m. / F 1450 p.p.m. with EDTA & $<0.0001$ & Significant & *D-G \\
F 1000 p.p.m. / F 0 p.p.m. with EDTA & $<0.0001$ & Significant & *D-H \\
F 1450 p.p.m. / F 5000 p.p.m. & $>0.9999$ & Ns & E-F \\
F 1450 p.p.m. / F 1450 p.p.m. with EDTA & $<0.0001$ & Significant & *E-G \\
F 1450 p.p.m. / F 0 p.p.m. with EDTA & $<0.0001$ & Significant & *E-H \\
F 5000 p.p.m. / F 1450 p.p.m. with EDTA & $<0.0001$ & Significant & *F-G \\
F 5000 p.p.m. / F 0 p.p.m. with EDTA & $<0.0001$ & Significant & *F-H \\
F 1450 p.p.m. with EDTA / F 0 p.p.m. & $>0.9999$ & Ns & G-H \\
with EDTA & & & \\
\hline
\end{tabular}

$H^{\prime}=0.2955 ; 5000$ p.p.m., $\left.H^{\prime}=0.2588\right)$. This suggested that the presence of $\mathrm{F}^{-}$favors the transformation of hydroxyapatite precursors, detected in the environment devoid of fluoride, to other calcium species. Under the condition containing $\mathrm{F}^{-}$, 
clustering of hydroxyapatite from the transitioning of other calcium species was detected as indicated by a decreased Shannon diversity index calculated from those groups treated with $\mathrm{F}^{-}$.

\subsection{Hydroxyapatite remineralization requires free $\mathrm{Ca}^{2+}$ in the remineralization condition}

As analyzed by XAS, the relative abundance of hydroxyapatite in the 1450 p.p.m. fluoride group and 1450 p.p.m. fluoride group with EDTA were compared to determine the role of $\mathrm{Ca}^{2+}$ in hydroxyapatite remineralization. The 1450 p.p.m. group with EDTA demonstrated a statistically significant difference $(P$-value $<0.0001)$ of $70.2 \%$ relative abundance of hydroxyapatite compared with $91.3 \%$ in the 1450 p.p.m. group (Table 2). In the environment containing 1450 p.p.m. fluoride, the diversity index $\left(H^{\prime}=0.2955\right)$ was lower than that of the calcium ion-deprived environment in the 1450 p.p.m. fluoride with EDTA $\left(H^{\prime}=0.8409\right)$. This discrepancy indicates that complete remineralization of hydroxyapatite requires free, unbound $\mathrm{Ca}^{2+}$ in the oral microenvironment in order to achieve the optimal aqueous condition needed for a remineralization to prevail.

\subsection{Contribution of $\mathrm{Ca}^{2+}$ and $\mathrm{F}^{-}$on the hydroxyapatite remineralization on early carious root dentin}

To elucidate the role of $\mathrm{Ca}^{2+}$ and $\mathrm{F}^{-}$in the remineralization process of carious root dentin, the relative abundance of hydroxyapatite in the acid-induced group and EDTA group were compared. It is worth noting that, in the absence of fluoride, $\mathrm{CaO}$ was detected and the diversity of calcium species was increased $\left(H^{\prime}=0.7120\right)$ when compared with the acid-induced group $\left(H^{\prime}=0.5803\right)$. In addition, there was no statistical significance $(P$-value $>0.05$, Table 2$)$ when comparing the percentage of hydroxyapatite in both groups, indicating that the relative abundance of the dominant calcium species remained unchanged in the absence of $\mathrm{F}^{-}$. Similar to the 1450 p.p.m. fluoride group with EDTA, there was no intense superficial band on the root formed in the 0 p.p.m. fluoride group with EDTA (Fig. 5). The percentage of hydroxyapatite regenerated in a 1450 p.p.m. fluoride group with EDTA (70.2\%) and a 0 p.p.m. fluoride group with EDTA $(69.3 \%)$ was not statistically different $(P$-value $>0.05$, Table 2$)$. However, a slight increase of the Shannon diversity index was found in a group of 0 p.p.m. fluoride with EDTA $\left(H^{\prime}=0.9367\right)$ when compared with a group of 1450 p.p.m. fluoride with EDTA $\left(H^{\prime}=0.8409\right)$. $\beta$-TCP was also present in a group of 0 p.p.m. fluoride with EDTA which lacked both $\mathrm{F}^{-}$and $\mathrm{Ca}^{2+}$, indicating that nucleation of calcium species on the root was diversified in the environment devoid of free $\mathrm{F}^{-}$and $\mathrm{Ca}^{2+}$.

The groups with 1000 p.p.m., 1450 p.p.m. and 5000 p.p.m. fluoride solutions were used to determine the effect of different concentrations of fluoride solutions on the relative abundance of remineralized hydroxyapatite. The relative abundance of hydroxyapatite across all three groups was statistically insignificant $(P$-value $>0.05)$ when compared with 0 p.p.m. fluoride (Table 2). Different concentrations of fluoride solutions had no effect on the relative abundance of remineralized hydroxyapatite, suggesting a robust effect of fluoride application on hydroxyapatite recrystallization, even at the 1000 p.p.m. fluoride concentration.

\section{Discussion}

Despite the significance of fluoride use as a non-invasive method in managing root carious lesions being extensively addressed (Cai et al., 2018), it is still controversial as to which concentration of fluoride is sufficient to achieve an optimal level of mineral regain. Thus, this is the first study investigating the fluoride-induced remineralization of hydroxyapatite by means of chemical structural analysis. Utilization of synchrotron radiation in dental biomaterial research has allowed us to closely elucidate the effect of fluoride in promoting hydroxyapatite crystal regeneration by means of a non-invasive strategy to gain a better understanding of how the mechanism of protection against root hydroxyapatite dissolution occurs in the presence of fluoride. In support of the use of synchrotron radiation, recent studies have demonstrated its potential for biomedical application including synchrotron radiation XAS for a precise cellular elemental localization as well as the identification of calcium compounds using XANES analysis in human cartilage (Nguyen et al., 2011; Ortega et al., 2009). Thus, an analysis of dental hard tissue and dental biomaterials using synchrotron radiation could be a laboratory strategy of choice that holds great promise for specific target structural detection with high sensitivity.

Acid produced by bacteria in dental plaque causes the dissolution of calcium and phosphate ions from apatite crystals (LeGeros, 1990). Our findings demonstrated the accumulation of $\mathrm{CaHPO}_{4}$ in addition to hydroxyapatite when root dentin was acid attacked. It is possible that a slight amount of hydroxyapatite was dissolved thereby increasing the deposition of $\mathrm{CaHPO}_{4}$ during an acid attack. Interestingly, TCP had not yet been recovered from dissolved hydroxyapatite possibly owing to the relatively higher solubility product constant $\left(K_{\mathrm{sp}}\right)$ of TCP species when compared with hydroxyapatite rendering hydroxyapatite the most stable form of calcium species examined (Chow, 2009). However, surface mineral loss is expected to have occurred in terms of quantity during the acid challenge (Wierichs et al., 2020). This is in part explained by a decreased deposition of hydroxyapatite in acidinduced specimens. Interestingly, the presence of $\mathrm{CaHPO}_{4}$ has suggested that it may possibly serve as a calcium reservoir required for hydroxyapatite nucleation on the root dentin.

Even though remineralization can occur even in the absence of fluoride in oral microenvironments (ten Cate, 1999), a treatment without $\mathrm{F}^{-}$resulted in a lower abundance of hydroxyapatite when compared with fluoride treatment. This suggested that $\mathrm{F}^{-}$specifically promotes hydroxyapatite remineralization of root dentin after a cariogenic attack. It is worth addressing that increasing concentrations of fluoride did not affect the relative abundance of hydroxyapatite formed. The relatively unchanged abundance of hydroxyapatite across environments with varying concentrations of fluoride 
was possibly due to the reached equilibrium between the remineralization of hydroxyapatite and the transformation of hydroxyapatite into fluoride species (Cury \& Tenuta, 2009; Li et al., 2014). Our $\mu \mathrm{CT}$ analysis also revealed a dense band of deposited mineral on the root when 1450 p.p.m. and 5000 p.p.m. fluoride were applied, possibly indicating the formation of fluoride-containing calcium species. However, higher fluoride content in the environments markedly decrease the size of crystallites (Asaizumi et al., 2017). Further quantification of fluoride-containing calcium species and characterization of the apatite crystal sizes are needed to corroborate the effect of fluoride use on fluorapatite deposition on root dentin in order to gain insight into how root dentin achieves resistance against acid challenge, due to the presence of a protective layer of acid-resistant fluoride species (McCann, 1968; Crommelin et al., 1983).

The loss of $\mathrm{Ca}^{2+}$ and $\mathrm{PO}_{4}{ }^{3-}$ is recovered when the biofilm is supersaturated with hydroxyapatite and fluorapatite at $\mathrm{pH}$ above 5.5 , suggesting that hydroxyapatite regeneration occurs concomitant with fluorapatite gain (Cury \& Tenuta, 2009). Our findings suggested that the absence of $\mathrm{F}^{-}$in a $\mathrm{Ca}^{2+}$-rich environment results in a redeposition of $\alpha$-TCP, a hydroxyapatite precursor, indicating that $\mathrm{F}^{-}$is required for promoting hydroxyapatite crystal maturation. When $\mathrm{Ca}^{2+}$ was removed by the EDTA chelation technique, a remineralization of hydroxyapatite failed to occur as indicated by the accumulation of $\alpha$-TCP, $\mathrm{CaHPO}_{4}$ and $\mathrm{CaO}$, which are known precursors of hydroxyapatite (Habibah \& Salisbury, 2020). Interestingly, $\mathrm{CaCO}_{3}$ was not detected which may suggest the dependence of hydroxyapatite recrystallization on the availability of $\mathrm{Ca}^{2+}$ and $\mathrm{PO}_{4}{ }^{3-}$ ions, not on carbonate ions. Perhaps carbonate apatite is formed but the high solubility of carbonate in an acidic microenvironment may lead to its absence on tooth substrates (Neel et al., 2016).

In an environment devoid of both $\mathrm{F}^{-}$and $\mathrm{Ca}^{2+}, \beta$-TCP was formed rather than $\alpha$-TCP. The lack of $\mathrm{F}^{-}$and $\mathrm{Ca}^{2+}$ might allow the shift of hydroxyapatite on root dentin to $\beta$-TCP, which is a more stable form when compared with $\alpha$-TCP (Cohn et al., 2017), to redeposit on root dentin. Undersaturation of $\mathrm{F}^{-}$in the milieu might thus fail to induce a complete hydroxyapatite recrystallization, and the effect was even more pronounced in the environment lacking both $\mathrm{F}^{-}$ and $\mathrm{Ca}^{2+}$. Despite the presence of $\mathrm{F}^{-}$, the lack of $\mathrm{Ca}^{2+}$ in the environment might result in the incomplete transformation of hydroxyapatite precursors, i.e. $\alpha$-TCP, $\mathrm{CaHPO}_{4}$ and $\mathrm{CaO}$ into mature hydroxyapatite crystals. Although root dentin consists of calcium-containing precursors of hydroxyapatite, our results indicate that remineralization on root dentin is enhanced by unbound $\mathrm{Ca}^{2+}$, and the transformation of existing calcium precursors on the root dentin to mature hydroxyapatite is driven by $\mathrm{F}^{-}$in the environment. Taken together, our data indicated that $\mathrm{Ca}^{2+}$ and $\mathrm{F}^{-}$in the environment contribute to hydroxyapatite nucleation. The clinical implications of this applies directly to xerostomic patients or patients receiving radiotherapy in the head and neck region, who may have limited salivary calcium and phosphate levels. This underscores the use of calcium-containing products such as casein phosphopeptide-amorphous calcium phosphate (CPP-ACP) in replenishing depleted oral calcium reservoirs, to possibly facilitate fluoride-mediated hydroxyapatite recrystallization (Rose, 2000). Use of fluoride-containing agents in conjunction with a topical CPP-ACP might allow a greater amount of hydroxyapatite recrystallization to occur in patients with high root caries risk, and also decreased toxicity when high dose fluoride is used.

In addition to hydroxyapatite, precursors of hydroxyapatite remained present as indicated in the aged root dentin group. A combination of $\beta$-TCP and fluoride are known to enhance the remineralization process (Arifa et al., 2019). This reaction can be used to explain the absence of TCP in the fluoridetreated samples where both $\mathrm{Ca}^{2+}$ and $\mathrm{F}^{-}$were present. This reaffirms that the presence of $\mathrm{F}^{-}$drives the transition of tricalcium phosphate into fluoride species (Li et al., 2014). The presence of $\mathrm{CaHPO}_{4}$, instead of TCP in the acid-induced and EDTA groups, can be attributed to the higher $K_{\text {sp }}$ of $\mathrm{CaHPO}_{4}$ (Chow, 2009). Herein, our laboratory analysis demonstrated a beneficial use of synchrotron radiation as an alternative method for dental biomaterial research analysis which could provide an accurate structural characterization under a highthroughput analysis using synchrotron-based XAS. Further investigation on formed fluoride-containing calcium species in the microenvironment containing salivary components in vivo is needed to provide insight on root mineral gain and aid in the rational use of fluoride to yield efficient root dentin protection under low concentration used. However, carbonated apatite structure has been identified and the nonstoichiometric forms of apatite are believed to be major components constituting human bone and teeth (Frank-Kamenetskaya, 2008; Leventouri et al., 2009). Further elucidation of the apatite-like structural deposition is needed for a better insight on natural remineralization of the nonstoichiometric apatite on root dentin as promoted by $\mathrm{F}^{-}$and $\mathrm{Ca}^{2+}$. To the best of our knowledge within the technical limitation of this study employing an artificial system, our results first however explained the dynamic basis of chemical hydroxyapatite dissolution from root dentin, in relation to its precursors. With hydroxyapatite being the dominant calcium species, understanding the dynamic change of hydroxyapatite remineralization in root dentin has implications on the prevention of root caries by targeting a specific mechanism required for maintaining structural homeostasis.

\section{Conclusion}

In summary, our study is the first laboratory quantitative report on the chemical gradient of calcium compounds associated with the remineralization of root dentin hydroxyapatite as analyzed by synchrotron radiation analysis - a novel qualitative approach. This study sheds light on the chemical aspect of hydroxyapatite remineralization on the root dentin by demonstrating that $\mathrm{F}^{-}$and $\mathrm{Ca}^{2+}$ are essential requirements and that the availability of calcium and phosphate ions is also vital for this process. Fluoride promotes a specific clustering of hydroxyapatite deposited on the root, regardless of the 
fluoride concentration used. The use of calcium phosphate products in tandem with fluoride for managing early root carious lesions is thus recommended.

\section{Acknowledgements}

We would like to thank Dr Benjamaporn Pitanuwat at the Department of Restorative Dentistry for a helpful discussion, Miss Chalermluck Phoovasawatscientist at the $\mu \mathrm{CT}$ beamline for aiding us in using the synchrotron facilities. Assistance on statistical analysis was kindly provided by Associate Professor Supaporn Chatchaiwiwattana, Department of Oral Biology.

\section{Funding information}

The following funding is acknowledged: KKU Faculty of Dentistry Research Funds 2019.

\section{References}

Arifa, M. K., Ephraim, R. \& Rajamani, T. (2019). Int. J. Clin. Pediatr. Dent. 12, 139-144.

Asaizumi, M., Yagi, N., Aoyama, K., Kato, T., Kuga, T., Oode, N., Oda, T., Sakurada, T., Nagase, S., Tabara, T. \& L. Karlinsey, R. (2017). Dent. Res. Management, pp. 20-30.

Beltrán-Aguilar, E. D., Barker, L. K., Canto, M. T., Dye, B. A., Gooch, B. F., Griffin, S. O., Hyman, J., Jaramillo, F., Kingman, A., Nowjack-Raymer, R., Selwitz, R. H. \& Wu, T. (2005). $M M W R$ Surveill. Summ. 54, 1-43.

Blumenthal, N. C. \& Posner, A. S. (1973). Calc. Tissue Res. 13, 235243.

Cai, J., Palamara, J. E. A., Manton, D. J. \& Burrow, M. F. (2018). Aust. Dent. J. 63, 34-54.

Cate, J. M. ten (1999). Acta Odontol. Scand. 57, 325-329.

Chaudhary, I., Tripathi, A. M., Yadav, G. \& Saha, S. (2017). Int. J. Clin. Pediatr. Dent. 10, 261-266.

Chow, L. C. (2009). Dent. Mater. J. 28, 1-10.

Cohn, M. R., Unnanuntana, A., Pannu, T. J., Warner, S. J. \& Lane, J. M. (2017). Comprehensive Biomaterials II, Vol. 7, pp. 278-297. Elsevier.

Crommelin, D. J., Higuchi, W. I., Fox, J. L., Spooner, P. J. \& Katdare, A. V. (1983). Caries Res. 17, 289-296.

Cury, J. A. \& Tenuta, L. M. A. (2009). Braz. Oral Res. 23, 23-30.

Dastmalchi, R., Poison, A., Bouwsma, O. \& Proskin, H. (1990). J. Clin. Periodontol. 17, 709-713.

Flaschka, H. \& Ganchoff, J. (1961). Talanta, 8, 720-725.

Francis, M. D. \& Webb, N. C. (1970). Calc. Tis Res. 6, 335-342.

Frank-Kamenetskaya, O. V. (2008). Minerals as Advanced Materials I, edited by S. V. Krivovichev, pp. 241-252. Berlin, Heidelberg: Springer.

Habibah, T. U. \& Salisbury, H. G. (2020). StatPearls, pp. 1-5.
Heasman, P. A., Ritchie, M., Asuni, A., Gavillet, E., Simonsen, J. L. \& Nyvad, B. (2017). J. Clin. Periodontol. 44, S178-S193.

Kantrong, N., Mongkontunpimon, W., Supameteeworakul, S. \& Wongkhantee, S. (2021). Odontology, 109, 124-138.

Keowmaneechai, E. \& McClements, D. J. (2002). J. Agric. Food Chem. 50, 7145-7153.

Kim, I. Y. \& Ohtsuki, C. (2016). Ceram. Int. 42, 1886-1890.

Klysubun, W., Sombunchoo, P., Deenan, W. \& Kongmark, C. (2012). J. Synchrotron Rad. 19, 930-936.

Lacy, J. (1963). Talanta, 10, 1031-1040.

LeGeros, R. Z. (1990). J. Dent. Res. 69, 566-567.

Leventouri, T., Antonakos, A., Kyriacou, A., Venturelli, R., Liarokapis, E. \& Perdikatsis, V. (2009). Int. J. Biomater. 2009, 1-6. Li, X., Wang, J., Joiner, A. \& Chang, J. (2014). J. Dent. 42, S12-S20. McCann, H. G. (1968). Arch. Oral Biol. 13, 987-1001.

Neel, E. A. A., Aljabo, A., Strange, A., Ibrahim, S., Coathup, M., Young, A. M., Bozec, L. \& Mudera, V. (2016). Int. J. Nanomedicine, 11, 4735-4741.

Nguyen, C., Ea, H. K., Thiaudiere, D., Reguer, S., Hannouche, D., Daudon, M., Lioté, F. \& Bazin, D. (2011). J. Synchrotron Rad. 18, 475-480.

Ortega, R., Devès, G. \& Carmona, A. (2009). J. R. Soc. Interface, 6, s649.

Ravel, B. \& Newville, M. (2005). J. Synchrotron Rad. 12, 537-541.

Reynolds, E. C. (2008). Aust. Dent. J. 53, 268-273.

Rose, R. K. (2000). Arch. Oral Biol. 45, 569-575.

SBU (2002). Preventing Dental Caries Stockholm. SBU Report No. 161. Swedish Council on Health Technology Assessment in Health. (In Swedish.)

Shahmoradi, M., Bertassoni, L. E., Elfallah, H. M. \& Swain, M. (2014). Adv. Calcium Phosphate Biomater. 2, 511-547.

Slayton, R. L., Urquhart, O., Araujo, M. W. B., Fontana, M., GuzmánArmstrong, S., Nascimento, M. M., Nový, B. B., Tinanoff, N., Weyant, R. J., Wolff, M. S., Young, D. A., Zero, D. T., Tampi, M. P., Pilcher, L., Banfield, L. \& Carrasco-Labra, A. (2018). J. Am. Dent. Assoc. 149, 837-849.e19.

Sobczak-Kupiec, A. \& Wzorek, Z. (2012). Ceram. Int. 38, 641-647.

Sui, T., Salvati, E., Harper, R. A., Zhang, H., Shelton, R. M., Landini, G. \& Korsunsky, A. M. (2018). Acta Biomater. 77, 333-341.

Tanaka, T., Yagi, N., Ohta, T., Matsuo, Y., Terada, H., Kamasaka, K., To-o, K., Kometani, T. \& Kuriki, T. (2010). Caries Res. 44, 253-259.

UN (2019). World Population Ageing 2019 Highlights. Report ST/ ESA/SER. A/444. New York: Department of Economic and Social Affairs of the United Nations.

Wierichs, R. J. \& Meyer-Lueckel, H. (2015). J. Dent. Res. 94, 261-271.

Wierichs, R. J., Rupp, K., Meyer-Lueckel, H., Apel, C. \& EstevesOliveira, M. (2020). Caries Res. 54, 75-86.

Young, D. A., Nový, B. B., Zeller, G. G., Hale, R., Hart, T. C., Truelove, E. L., Ekstrand, K. R., Featherstone, J. D. B., Fontana, M., Ismail, A., Kuehne, J., Longbottom, C., Pitts, N., Sarrett, D. C., Wright, T., Mark, A. M. \& Beltran-Aguilar, E. (2015). J. Am. Dent. Assoc. 146, 79-86.

Zipkin, I. (1970). Biological Calcification: Cellular and Molecular Aspects, pp. 69-103. Boston: Springer, Boston, MA. 